\title{
An empirical spectral library of chemically well characterized stars for stellar population modelling
}

\author{
André de Castro Milone ${ }^{1}$, Anne E. Sansom ${ }^{2}$, Patricia \\ Sánchez-Blázquez ${ }^{3}$, Alexandre Vazdekis ${ }^{4,5}$, Jesus Falcón-Barroso ${ }^{4,5}$ \\ and Carlos Allende Prieto ${ }^{4,5}$ \\ ${ }^{1}$ Divisão de Astrofísica, Instituto Nacional de Pesquisas Espaciais, \\ Av. dos Astronautas 1758, São José dos Campos, SP 12227-010, Brazil \\ ${ }^{2}$ Jeremiah Horrocks Institute, University of Central Lancashire, Preston, Lancs PR1 2HE, UK \\ ${ }^{3}$ Departamento de Física Teórica, Universidad Autónoma de Madrid, Cantoblanco 28409, \\ Madrid, Spain \\ ${ }^{4}$ Instituto de Astrofísica de Canarias, Vía Láctea s/n, E-38205 La Laguna, Tenerife, Spain \\ ${ }^{5}$ Departamento de Astrofísica, Universidad de La Laguna, E-38205 La Laguna, Tenerife, Spain \\ email: andre.milone@inpe.br
}

\begin{abstract}
With the goal of assembling a new generation of more realistic single stellar population (SSP) models, we have obtained magnesium abundances for nearly $80 \%$ of the stars of the widely employed MILES empirical spectral library. Additional spectroscopic observations of carefully selected stars have recently been obtained to improve the parametric coverage of this library. Here we report on: (i) the framework of $\mathrm{Mg}$ abundance determination carried out at mid-resolution, (ii) the newly acquired data, and (iii) the preliminary steps towards modelling stellar populations.
\end{abstract}

Keywords. astronomical data bases: miscellaneous, stars: abundances, stars: atmospheres, techniques: spectroscopic.

\section{Introduction}

One limitation of current stellar population models is the use of nearby stars whose atmospheres are not completely characterized in terms of elemental abundances. Typically, these models take the iron abundance as a metallicity tracer, but stellar spectra may change considerably if the abundance ratios between iron and other metals depart from the scaled-solar mixture. Furthermore, stars from Galaxy's distinct components show different abundance patterns.

Accounting for various elemental abundances, including alpha (traced by $\mathbf{M g}$ ) and iron peak elements (traced by $\mathbf{F e}$ ), will allow us to provide an empirical stellar library that is particularly useful for modelling spectral energy distributions of evolving stellar populations. Whilst $\mathrm{Mg}$ is produced via $\mathrm{C}$ burning in massive stars and ejected into the interstellar medium mainly through Type II supernovae (SNe), Fe is mainly released in Type Ia SNe. The higher the $\mathbf{M g} / \mathbf{F e}$ abundance ratio, the shorter the time scale of star formation (see, e.g., McWilliam 1997). 


\section{The MILES library}

The MILES data base contains good-quality flux-calibrated optical spectra for 985 stars with a uniform FWHM resolution of $2.5 \AA$ (Sánchez-Blázquez et al. 2006, Falcón-Barroso et al. 2011). This library presents an unprecedented coverage of stellar parameters. In Cenarro et al. (2007) the main photospheric parameters of individual stars were carefully transformed from data of several high-resolution spectroscopic analyses into a homogeneous system with great accuracy $\left(\sigma \mathrm{T}_{\text {eff }}=100 \mathrm{~K}, \sigma \log \mathrm{g}=0.2, \sigma[\mathrm{Fe} / \mathrm{H}]=0.1\right.$ dex $)$. MILES is regarded nowadays as a standard empirical stellar library, and it is publically available through a user-friendly website (http://miles.iac.es).

\section{The MILES Mg/Fe Catalogue}

$[\mathrm{Mg} / \mathrm{Fe}]$ abundance ratios were collated from high-resolution $(H R)$ studies and transformed onto a uniform scale having the wide catalogue of Borkova \& Marsakov (2005, hereafter BM2005) as reference. Milone, Sansom \& Sánchez-Blázquez (2011) also carried out a spectroscopic analysis at mid-resolution $(\mathrm{mr})$ to extend the $\mathrm{Mg}$ characterization by applying spectral synthesis on the MILES spectra with the MOOG LTE code (Sneden 2002, http://verdi.as.utexas.edu) through an automatic process excluding poorly matched cases. To calibrate our mr measurements we used a control sample with 309 library's stars (255 dwarfs, being 219 from BM2005, and 51 giants from $H R$ works).

Two strong Mg I features (at $\lambda 5183.60 \AA$ and $\lambda 5528.40 \AA$ ) were analysed through the pseudo-equivalent width and line profile fit methods. We linearly interpolated model atmospheres over the MARCS 2008 grid (Gustafsson et al. 2008) that suitably follow the general chemical pattern of our Galaxy for the $\alpha$-elements. We adopted accurate atomic transition data from VALD and molecular line data compiled by Kurucz (1995).

The catalogue provides data for 752 MILES stars (411 dwarfs \& 341 giants) identifying their sources. The $\sigma[\mathrm{Mg} / \mathrm{Fe}]$ weighted average is $\sim 0.1$ dex being 0.09 and 0.12 dex for the $H R$ and $m r$ measurements respectively. The abundance ratio distribution is fairly flat across the MARCS models' parameter space. The $H R$ data are spread along the main-sequence basically from $\mathrm{T}_{\text {eff }} \approx 4500 \mathrm{~K}$ up to around $10000 \mathrm{~K}$ and on the giant branch mainly from $4000 \mathrm{~K}$ up to $5500 \mathrm{~K}$. Our $\mathrm{mr}$ measurements have a wide distribution over the Herztsprung-Russell diagram, however there are deficiencies in the low mainsequence, red giant branch tip and hottest giants. The $\mathrm{mr}$ data statistically recover with acceptable accuracy the solar neighbourhood global pattern of $[\mathrm{Mg} / \mathrm{Fe}]$ as a function of $[\mathrm{Fe} / \mathrm{H}]$ traditionally defined by $H R$ data.

\section{New data set for MILES}

We have compiled from $H R$ works a new set of candidate stars to be incorporated into the MILES library. The whole set contains around one thousand stars spread over both sky hemispheres by adopting as reference the PASTEL stellar parameter catalogue (Soubiran et al. 2010). The motivation is to improve the sample distribution over the MILES 4-D parameter space $\left(\mathrm{T}_{\text {eff }}, \log \mathrm{g},[\mathrm{Fe} / \mathrm{H}],[\mathrm{Mg} / \mathrm{Fe}]\right)$. Having excluded spectroscopic binaries, anomalous variable and peculiar stars, we have defined a sample of around 400 field stars that are observable with the Isaac Newton Telescope (INT) at Observatorio del Roque de Los Muchachos (Canary Islands, Spain).

We have observed this year 218 new stars in total using the same spectroscopic instrumental setup employed for the original MILES library (INT with the Intermediate Dispersion Spectrograph). The photospheric parameters and $\mathrm{Mg}$ abundances of the new sample stars have yet to be calibrated to the MILES system. 


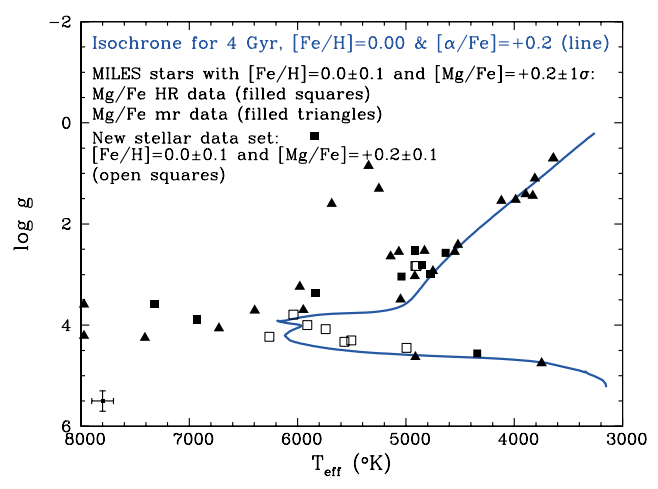

Figure 1. Selected set of MILES stars versus an isochrone from Dotter et al. (2008).

\section{SSP modelling}

Unlike the Vazdekis et al. (2010) models, which show a Mg/Fe scaled-solar abundance ratio at solar metallicity but increase it with decreasing metallicity following the abundance pattern of solar neighbourhood, we intend to construct $\alpha$-enhanced models with variable $[\alpha / \mathrm{Fe}]$ for a range in ages and metallicities. We will build up state-of-the-art SSP models with distinct $\alpha / \mathrm{Fe}$ by selecting MILES stars according to their $[\mathrm{Mg} / \mathrm{Fe}]$ values within a 4-D intepolation scheme, whose spectra will be integrated along isochrones of similar $[\alpha / \mathrm{Fe}]$ under a self-consistent approach. Figure 1 exemplifies how the matching of MILES stars with an isochrone will be improved by including the new data set.

\section{Summary}

We have obtained $[\mathrm{Mg} / \mathrm{Fe}]$ with a precision of $\sim 0.1$ dex for about $80 \%$ of MILES that are placed on a uniform scale (available on request or in Milone et al. 2011). MILES is currently being expanded in around $20 \%$ through the inclusion of new stellar data set that fills in some gaps of the library 4-D parameter space and increase the star density in other regions. We will be able to compute a set of self-consistent semi-empirical SSP models with variable $\alpha$-enhancement for a range in ages and metallicities around solar, which will represent a benchmark for models based on theoretical atmospheres.

\section{Acknowledgements}

Milone thanks IAU and the Brazilian foundation Capes (AEX 3185/11-7). We thank R. Cacho, I. Martín and E. Mármol-Queraltó who helped us in one of the INT runs.

\section{References}

Borkova, T. V. \& Marsakov, V. A. 2005, AZh, 82, 453

Cenarro, J., Peletier, R. F., Sánchez-Blázquez, P., et al. 2007, MNRAS, 374, 664

Dotter, A., Chaboyer, B., Jevremović, D., et al. 2008, ApJS, 178, 89

Falcón-Barroso, J., Sánchez-Blázquez, P., Vazdekis, A., et al. 2011, A\&A, 532A, 95F

Gustafsson, B., Edvardsson, B., Eriksson, K., et al. 2008, A\&A, 486, 951

Kurucz, R. 1995, An Atomic and Molecular Data Bank for Stellar Spectroscopy, SAO, Cambridge McWilliam, A. 1997, ARAA, 35, 503

Milone, A. de C., Sansom, A. E., \& Sánchez-Blázquez, P. 2011, MNRAS, 414, 1227

Sánchez-Blázquez, P., Peletier, R. F., Jiménez-Vicente, J., et al. 2006, MNRAS, 371, 703

Soubiran, C., Le Campion, J.-F., Cayrel de Strobel, G., \& Caillo, A. 2010, A\& A, 515, 111

Vazdekis, A., Sánchez-Blázquez, P., Falcón-Barroso, J., et al. 2010, MNRAS, 404, 1639 